\title{
Advances in analytical chemistry
} using the unique properties of ionic liquids

\author{
Zhi-qiang Tan, Jing-fu Liu, Long Pang
}

Ionic liquids (ILs) are regarded as non-molecular solvents, as they are composed entirely of cations and anions. ILs possess several excellent unique properties (e.g., low volatility, high thermal stability, specific electrochemical characteristics, easy design, tunable viscosity, and miscibility with water or organic solvents). These properties make ILs attractive candidates for various analytical applications, the number of publications on which has increased exponentially in the past decade.

This article presents an overview of representative applications of ILs in advances in analytical chemistry that benefited from the unique properties of ILs, including the development achieved by using ILs as extraction solvents, dissolution solvents and separation media.

(๖) 2012 Elsevier Ltd. All rights reserved.

Keywords: Chromatographic separation; Dissolution; Electrochemical characteristics; Extraction; lonic liquid (IL); Microextraction; Miscibility; Separation; Task-specific extraction; Tunable viscosity

\footnotetext{
Zhi-qiang Tan, Jing-fu Liu*, Long Pang, State Key Laboratory of Environmental Chemistry and Ecotoxicology, Research Center for Eco-Environmental Sciences, Chinese Academy of Sciences, P.O. Box 2871, Beijing 100085, China
}

${ }^{*}$ Corresponding author. Fax: +86 10 62849192; E-mail: jfliu@rcees.ac.cn

\section{Introduction}

Room-temperature ionic liquids (ILs) are composed only of positively and negatively charged ions, and exist as liquids at relatively low temperatures $\left(<100^{\circ} \mathrm{C}\right)[1]$. Almost all of the ILs have been composed of organic cations and various anions. Typical cations are asymmetrically substituted nitrogen-containing compounds (e.g., imidazolium, pyridinium, quaternary ammonium, and the quaternary phosphonium group). Both inorganic anions (e.g., $\mathrm{Cl}^{-}, \mathrm{Br}^{-},\left[\mathrm{BF}_{4}\right]^{-},\left[\mathrm{PF}_{6}\right]^{-}$) and organic anions (e.g., trifluoromethylsulfonate $\left[\mathrm{CF}_{3} \mathrm{SO}_{3}\right]^{-}$, bis[(trifluoromethyl) sulfonyl]imide $\left[\left(\mathrm{CF}_{3} \mathrm{SO}_{2}\right)_{2} \mathrm{~N}\right]$, trifluoroethanoate $\left.\left[\mathrm{CF}_{3} \mathrm{CO}_{2}\right]^{-}\right)$are able to serve as the anionic counterparts [2,3]. As reported, theoretically, available combinations of cations and anions can give rise to as many as $10^{18}$ kinds of IL [4].

In contrast to common organic solvents, ILs have several outstanding properties (e.g., negligible vapor pressure, good thermal stability, wide electrochemical windows, intrinsic conductivity, excellent design, and varying solvation interaction) [5]. Due to these characteristics, ILs are involved in a lot of fields \{e.g., synthesis
[6], catalysis [7], chemical industry [8] and analytical chemistry\} to compensate for the limitations of classical organic solvents. The unique properties of ILs give rise to a great number of applications in analytical chemistry, and comprehensive review articles on this field have been published [2,3,9-14]. In these reviews, ILs were proposed as promising materials to address the various challenges within the scope of analytical chemistry. Nevertheless, it is of great interest to comment explicitly on the significant advances in analytical chemistry resulting from the application of ILs. Table 1 lists the structures, the properties and the applications of a few representative ILs.

The goal of this review is to highlight the advances using ILs in analytical chemistry benefiting from the unique properties of ILs. We try to overview this progress in the major sub-disciplines of analytical chemistry, including extraction, separation, characterization and detection. Due to the large amounts of publications related to this topic, we do not attempt to refer all the publications, and virtually only the more representative reports are selected for conciseness. 


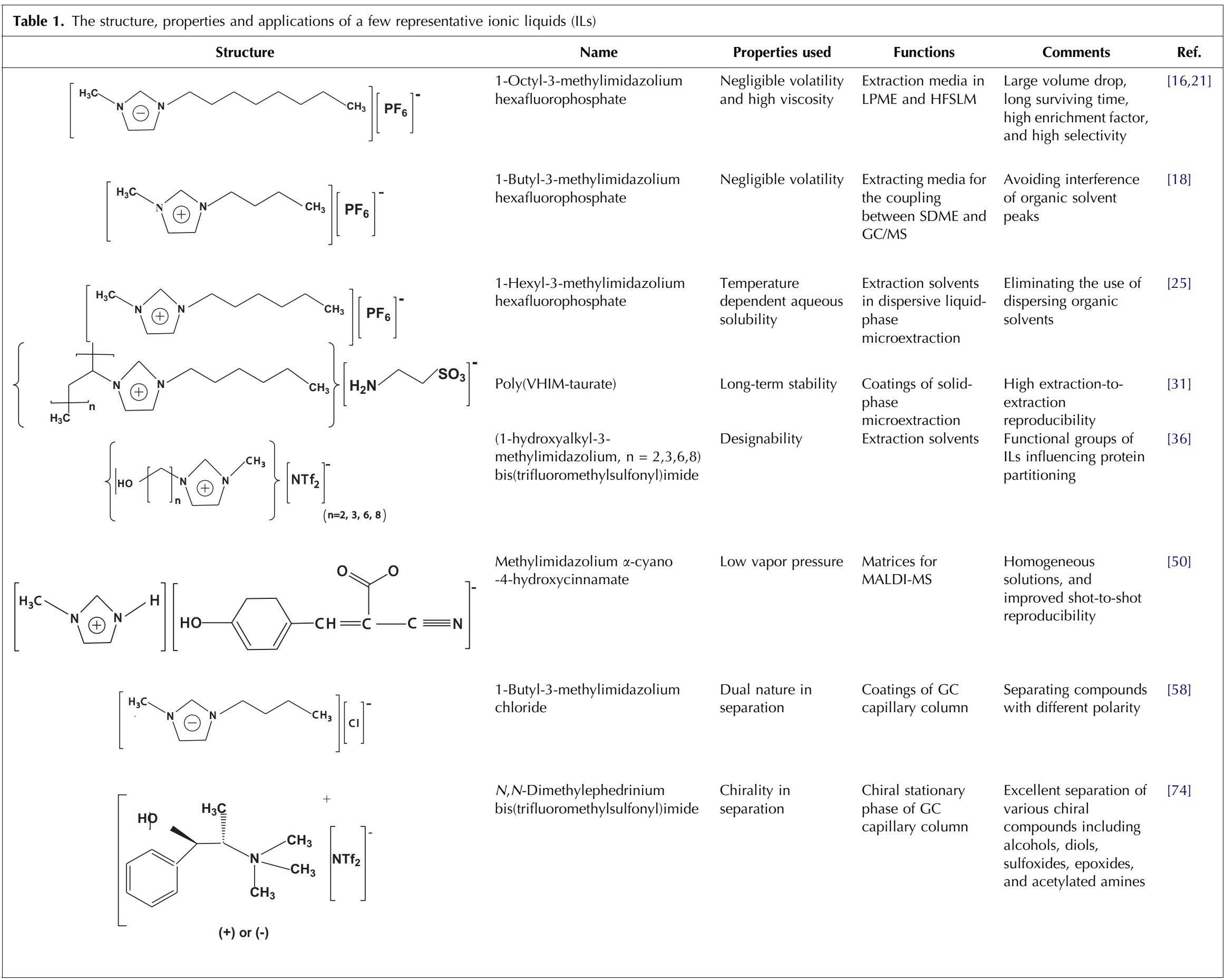




\section{Advance in analytical chemistry with ILs as extraction solvent}

\subsection{Microextraction}

Traditional liquid-liquid extraction (LLE) suffered from the use of large amounts of volatile and toxic organic solvents that have adverse effects on the operators and environment. To overcome this limitation, liquid-phase microextraction (LPME) and solid-phase microextraction (SPME) that use no or very small amounts of organic solvents were developed. LPME like single-drop microextraction (SDME) [15] reduces the consumption of organic solvent to a few mL, which effectively avoids the deleterious effects of hazard organic solvents on humans and the environment. However, due to the high volatility and low viscosity of traditional organic solvents (e.g., toluene and 1-octanol), the drop volume and the sustainable operation time of headspace extraction are very limited, giving rise to the small amounts of analytes extracted and thus low method sensitivity.

The unique properties of non-volatility and adequate viscosity mean that ILs are promising substitutes for traditional organic solvents in SDME. Our study [16] demonstrated that ILs \{e.g., 1-octyl-3-methylimidazolium hexafluorophosphate $\left.\left(\left[\mathrm{C}_{8} \mathrm{MIM}\right]\left[\mathrm{PF}_{6}\right]\right)\right\}$ are excellent extraction solvents in both direct-immersion and headspace SDME. In comparison to 1-octanol, a larger volume drop of $\left[\mathrm{C}_{8} \mathrm{MIM}\right]\left[\mathrm{PF}_{6}\right]$ can be formed and survive for a longer extraction time, so a much higher enrichment factor for polycyclic aromatic hydrocarbons (PAHs) is achieved. While direct-immersion SDME provides a higher enrichment factor than headspace SDME for less volatile PAHs, the enrichment factor obtained by headspace SDME was almost three-fold that by direct-immersion SDME in 30-min extraction of the most volatile PAHs, indicating that headspace SDME is superior to directimmersion SDME when volatile analytes are extracted.

Further study demonstrated that the non-volatile, thermostable properties of ILs make them ideal solvents for headspace SDME of semi-volatile compounds at an elevated temperature [17]. For example, $\left[\mathrm{C}_{4} \mathrm{MIM}\right]\left[\mathrm{PF}_{6}\right]$ showed excellent extraction efficiency for headspace SDME of chloroanilines in environmental water samples at increased temperature. Because increased temperature usually improves the evaporation of target compounds from the sample matrix to the headspace, the increase of temperature from $50^{\circ} \mathrm{C}$ to $90^{\circ} \mathrm{C}$ gave rise to about 4-6 times enhancement of the extraction efficiency for the four chlorinated anilines studied.

While the negligible volatility of ILs favors headspace SDME, this same property of ILs make them incompatible with direct coupling of IL-based SDME with gas chromatography (GC), as the injection of ILs into GC could contaminate the GC column. This obstacle was overcome with the development of a removable interface that permits the direct injection of the ILs into GC [18]. A cotton-containing tube in this interface enables the transfer of the extracted analytes from the IL microdroplet to the separation column while preventing the IL from entering and contaminating the column. Using this interface, dichloromethane, $p$-xylene, and n-undecane were determined by coupling $\left[\mathrm{C}_{4} \mathrm{MIM}\right]\left[\mathrm{PF}_{6}\right]$-based SDME with GC-MS. It is noteworthy that this system required no solvent delay, so polar compounds with short retention time could be recorded and quantitatively determined. The non-volatility and thermostability avoided the interference of highly intense organic solvent peaks, allowing the determination of analytes with different polarities and boiling points.

Although hydrophobic ILs \{e.g., $\left.\left[\mathrm{C}_{8} \mathrm{MIM}\right]\left[\mathrm{PF}_{6}\right]\right\}$ were applicable for direct-immersion SDME, their dissolution prohibited their use in sampling large volumes of aqueous solutions and long-term extraction, which caused dissolution or loss of the ILs and therefore low method sensitivity. To overcome this problem, Anderson et al. [19] introduced a new class of ILs containing the tris(pentafluoroethyl)trifluorophosphate (FAP) anion paired with the imidazolium, phosphonium or pyrolidinium cation. In comparison to other hydrophobic ILs containing the $\left[\mathrm{PF}_{6}\right]^{-}$and bis[(trifluoromethyl)sulfonyl]imide $\left(\mathrm{NTf}_{2}{ }^{-}\right)$anions, these FAP-based ILs are more hydrophobic and hydrolytically stable, permitting them to be used in the sampling of large volumes of aqueous solutions without dissolution or loss of the IL, thereby providing larger enrichment factors for a variety of analytes, including PAHs.

As another commonly used mode of LPME, hollowfiber-supported liquid-membrane extraction (HFSLME) is very useful for extracting weak organic acids and bases with high selectivity and enrichment factor [20]. However, there is a dilemma in selecting the liquid membrane for HFSLME of hydrophilic compounds. According to the "like dissolves like" principle, polar organic solvents (e.g., dichloromethane) are favored for use as liquid membranes for extracting hydrophilic compounds. However, due to their high water solubility and volatility, organic solvents with high polarity are unsuitable for use as membrane liquid, and, as a compromise, relatively low polar solvents (e.g., 1-octanol and dihexyl ether) have to be used as the extraction solvent in HFSLME. This usually sacrifices extraction efficiency. Thus, finding novel solvents that are polar and non-volatile for HFSLME is of great interest. Given its high affinity to polar compounds and low volatility/water solubility, $\left[\mathrm{C}_{8} \mathrm{MIM}\right]\left[\mathrm{PF}_{6}\right]$ was used as membrane liquid for HFSLME of chlorophenols in environmental water samples [21]. Further study indicated that, while conventional membrane liquids (e.g., undecane, 1-octanol and dihexyl ether) are inefficient in extraction of amphoteric sulfonamide antibiotics, $\left[\mathrm{C}_{8} \mathrm{MIM}\right]\left[\mathrm{PF}_{6}\right]$ showed promising 
extraction efficiency due to its high affinity to both nonpolar and polar compounds [22]. For all five sulfonamides studied, the enrichment factor with $\left[\mathrm{C}_{8} \mathrm{MIM}\right]\left[\mathrm{PF}_{6}\right] /$ $4 \%$ trioctylphosphine oxide (TOPO) as liquid membrane was about twice that with DHE/4\% TOPO.

Dispersive liquid-liquid microextraction (DLLME) is one of the most attractive LPME modes due to its simplicity and speed [23]. As the water solubility of some ILs strongly depends on temperature, the mixture of an IL with water forms two phases at lower temperature and a homogeneous phase at high temperature, which is quite meaningful to build an IL-water biphasic system [24]. Based on this phenomenon of ILs, Zhou et al. [25] developed controlled DLLME. Unlike traditional DLLME, the dispersing was not realized by injecting any organic solvents but by the change of temperature. After extraction at high temperature, the IL concentrating with analytes was concentrated into one drop simply by cooling and centrifugation.

The near non-volatility of ILs was also exploited in SPME, which is a widely used sample-preparation technique developed by Arthur and Pawliszyn in the early 1990s [26]. Compared to SDME, SPME has the advantages of being robust and having a larger surface-tovolume ratio. Liu et al. [27] for the first time developed a disposable $\left[\mathrm{C}_{8} \mathrm{MIM}\right]\left[\mathrm{PF}_{6}\right]$-coated headspace SPME fiber for headspace extraction of benzene, toluene, ethylbenzene, and xylenes (BTEX) in paints. This method was improved by Hsieh et al. [28] by coating a film of Nafion on the fiber prior to the coating of ILs, which enhanced the amount and the stability of IL film adsorbed on the fiber. In the above two procedures, IL coatings were physically adsorbed on the surface of a fused-silica capillary tubing or a stainless-steel fiber, which led to the IL coatings coming off and therefore the requirement to recoat the fiber after each extraction.

To overcome those drawbacks, Anderson and coworkers designed a series of polymeric ILs (PILs) for use as coatings of SPME [29-31]. These PIL-based coatings showed long-term stability and high extraction-toextraction reproducibility, and were successfully used for selective extraction of hydrocarbons and fatty acid methyl esters with high boiling points and low vapor pressures. To lessen the bleeding problem, Armstrong's group [32] synthesized two ILs (dicationic ILs and styrene-based PILs) and bonded them onto silica particles for use as adsorbent in SPME. Both headspace and immersion models of SPME were attempted to gauge the capabilities of the bonded IL sorbents for extracting small, polar molecules. Results demonstrated that stability of SPME fibers coated with polymerized IL-bonded silica particles was greatly improved.

He et al. [33] proposed a new approach to increase the thermal stability and lifetime of IL-based SPME fiber coatings. By crosslinking an IL-impregnated silicone elastomer on a fused-silica support, they generated reusable IL-based SPME fibers with enhanced thermal stability and fiber lifetime for the determination of methamphetamine and amphetamine in human urine.

Very recently, our group fabricated an SPME fiber by coating stainless-steel fiber with a PIL through covalent bonding. The stainless-steel fiber was sequentially coated with a gold film, a monolayer of 3-(mercaptopropyl) triethoxysilane on the gold layer, and a silica layer. 1-Vinyl-3-(3-triethoxysilylpropyl)-4,5-dihydro-imidazolium chloride IL was anchored on the silica layer by covalent bond, and the PIL film was further formed by free radical copolymerization between 1-vinyl-3-(3triethoxysilylpropyl)-4,5-dihydroimidazdium and vinylsubstituted imidazolium with azobisisobutyronitrile (AIBN) as initiator. This PIL fiber exhibited comparable analytical performance to a commercial PDMS fiber in extracting PAHs [34].

\subsection{Task-specific extraction}

ILs comprise a class of solvents that can be designed and synthesized based on the specific requirement. This property is very useful to develop ILs for task-specific extraction (e.g., the highly selective extraction of specific analytes).

Armstrong's group [35] found that compounds containing acidic functional groups (e.g., carboxyl and phenolic hydroxyl) partitioned less to the $\left[\mathrm{C}_{4} \mathrm{MIM}\right]\left[\mathrm{PF}_{6}\right]$ phase than to octanol, amine-group-bearing compounds exhibited higher partitioning coefficients to the $\left[\mathrm{C}_{4} \mathrm{MIM}\right]\left[\mathrm{PF}_{6}\right]$ phase than to octanol, and the others (neutral molecules and ionizable compounds with both basic and acidic functions) exhibited similar distribution behavior in both systems. The two studies above implied that it is possible to design specific ILs for selective extraction of target organic compounds.

Lys-rich protein cytochrome $\mathrm{c}$ was quantitatively extracted into hydroxyl group-containing ILs with dicyclohexano-18-crown-6 (DCH18C6) through the formation of supramolecular complexation. It was demonstrated that the hydrophobicity and functional groups of ILs have a great influence on protein partitioning, and a hydroxyl group-containing IL with DCH18C6 is capable of quantitative extraction of cytochrome c [36].

Further, the same group developed a new task-specific IL $\left\{[18 \mathrm{C} 6 \mathrm{MIM}]\left[\mathrm{PF}_{6}\right]\right\}$ by attaching the crown-ether moiety to the imidazolium cation, and a hydroxyethyl group containing IL $\left\{\left[\mathrm{C}_{2} \mathrm{OHMIM}\right]\left[\mathrm{Tf}_{2} \mathrm{~N}\right]\right\}$. The mixture prepared by dissolving $[18 \mathrm{C} 6 \mathrm{MIM}]\left[\mathrm{PF}_{6}\right]$ in $\left[\mathrm{C}_{2} \mathrm{OH}-\right.$ $\mathrm{MIM}]\left[\mathrm{Tf}_{2} \mathrm{~N}\right]$ provided similar extraction behavior to that using DCH18C6 as the extractant [37].

Functional amino-acid ILs were used as solvent and selector in chiral LLE of the L-enantiomer of amino acid from a mixture of L-enantiomer and D-enantiomer in ethyl acetate [38].

In extraction of metal ions with conventional organic solvents, additional organic and inorganic complex 
agents are usually introduced to improve their extraction efficiencies. For the purpose of extracting $\mathrm{Hg}^{2+}$ and $\mathrm{Cd}^{2+}$ into the IL phase from water without the addition of additional complex agents, Visser et al. [39] designed and synthesized a series of hydrophobic task-specific ILs by incorporating urea-, thiourea- and thioether-substituted alkyl groups to imidazoles. They demonstrated that these task-specific ILs showed enhanced distribution ratios for metal ions compared to ILs without the substitution groups, and ILs containing thiourea- and urea-derivatized cations provided higher distribution ratios for $\mathrm{Hg}^{2+}$ than for $\mathrm{Cd}^{2+}$.

Recently, it was reported that trifluoroacetylacetonefunctionalized imidazolium-based ILs loaded onto silica particles showed fast and almost quantitative (>99.9\%) extraction of lanthanide salts $(\mathrm{Eu}, \mathrm{Tb})$ from dilute aqueous solution of $\mathrm{NaOH}$ as their diketonato complexes. The procedure is reproducible and the loaded $\mathrm{SiO}_{2}$ particles can be simply treated with acid to strip the metal ions and regenerate the adsorbed (protonated) extractant [40].

The 2-butylthiolonium bis(trifluoromethanesulfonyl)amide ([MIMSBu] $\left[\mathrm{NTf}_{2}\right]$ ) was developed for the selective extraction of $\mathrm{Cu}$ (II) from aqueous solution. $[\mathrm{MIMSBu}]\left[\mathrm{NTf}_{2}\right]$ functioned as both extraction agent and solvent for $\mathrm{Cu}(\mathrm{II})$, so, in this extraction process, no complexing agent was required. Furthermore, [MIMSBu] $\left[\mathrm{NTf}_{2}\right]$ can be recovered from the labile copper-water-IL interacting system by washing with a strong acid. High selectivity of $\mathrm{Cu}$ (II) extraction is achieved relative to that of other divalent transition-metal cations [e.g., Co(II), $\mathrm{Fe}(\mathrm{II})$, and $\mathrm{Ni}(\mathrm{II})]$ [41].

The designable property of ILs also provides great opportunities for the development of task-specific SPME fibers. Amine-functionalized ILs are capable of sequestering $\mathrm{CO}_{2}$ as a carbamate salt [42]. Based on this property, two task-specific PILs [i.e. poly(1-vinyl-3hexylimidazolium) taurinate and poly(1-vinyl-3-hexylimidazolium) $\mathrm{NTf}_{2}$ ] with different selectivities toward $\mathrm{CO}_{2}$ were developed for use as sorbent coatings. The poly(1-vinyl-3-hexylimidazolium)- $\mathrm{NTf}_{2}$ fiber was found to possess superior selectivity of $\mathrm{CO}_{2} / \mathrm{CH}_{4}$ and $\mathrm{CO}_{2} / \mathrm{N}_{2}$ compared to the commercially available Carboxen fiber [43].

\subsection{Micowave-assisted extraction (MAE)}

Compared with traditional organic solvents, ILs are much more efficient in absorbing and transferring microwave energy [44]. Therefore, microwave-assisted extraction (MAE) in ILs showed higher extraction efficiency within less extraction time than in traditional organic solvents [45].

With aqueous solution of $\left[\mathrm{C}_{4} \mathrm{MIM}\right] \mathrm{Br}$ as MAE solvent, trans-resveratrol was extracted from Chinese traditional medicinal herb Rhizma polygoni cuspidati [46]. Similarly, PAHs were extracted from sediments by MAE with aqueous solutions containing aggregates of the $\left[\mathrm{C}_{16} \mathrm{MIM}\right] \mathrm{Br}$ as the extracting medium [47].

Combining MAE with SDME, a new method termed microwave-assisted headspace single-drop microextraction was also developed for the extraction of chlorobenzenes from water samples [48].

\section{Advances in analytical chemistry with ILs as dissolution solvents}

Because of their ultra-low volatility and the excellent solubility for various analytes, ILs have been adopted as dissolution solvents or matrices for quartz-crystal microbalance (QCM) sensors, headspace GC, matrix-assisted laser desorption/ionization mass spectrometry (MALDI-MS) analysis, NMR analysis, as well as vis/NIR and Raman spectroelectrochemistry. Given these applications of ILs have been well discussed in previous review papers, we remark on only a few representative advances.

\subsection{Matrix-assisted laser desorption/ionization mass spectrometry}

MALDI is frequently employed to determine both large biological and synthetic molecules. The ideal matrix should not only contain a chromophore to absorb laser light for ionization, but also be non-volatile under highvacuum conditions. Traditional solid matrices suffer from irreproducibility as a result of the solute and impurities segregating in them. By contrast, ILs are ideal matrices for MALDI-MS as they produce a much more homogeneous sample solution yet have greater vacuum stability than most solid matrices. Their low vapor pressure makes ILs remain liquid, and permits them to keep the status even in a vacuum [49], which could enhance method reproducibility and sensitivity.

Armstrong's group is the first and most active in studying the use of ILs as matrices for MALDI-MS. They found that ILs apt to donate free $\mathrm{H}^{+}$are effective candidates as matrices for MALDI-MS, whereas most conventional ILs without this property are ineffective as MALDI matrices [50]. Later, they discovered that both proton affinity and pKa of the cation strongly impact the ability of IL matrices to ionize the analytes. ILs can be used to detect polysaccharides with fewer degradation products than solid matrices. $N, N$-diisopropylethylammonium $\alpha$ cyano-4-hydroxycinnamate and $\mathrm{N}$-isopropyl- $\mathrm{N}$-methyl$t$-butylammonium $\alpha$-cyano-4-hydroxycinnamate were the best matrices for proteins and peptides, whereas $N$, $N$-diisopropylethylammonium $\alpha$-cyano-4-hydroxycinnamate and $N, N$-diisopropylethylammonium ferulate were the best matrices for carbohydrates. [51]. Also, N,Ndiisopropylethylammonium $\alpha$-cyano-4-hydroxycinnamate (DEA-CHCA) was developed for the characterization of polar biodegradable polymers with enhanced signal 
and minimized polymer degradation, allowing accurate, sensitive determination of the number average molecular weights, the weight average and the polydispersity index of labile polar polymers [52].

MS imaging (MSI) is a powerful tool for the direct detection of biomolecules (e.g., phospholipids, proteins and peptides) in tissue samples. Currently, most MSI studies are conducted with a MALDI or secondary-ion MS (SIMS) ion source coupled to a time-of-flight (TOF) mass analyzer [53]. An IL matrix composed of $\alpha$-cyano-4-hydroxy-cinnamic acid (CHCA) and 1-methylimidazole $(1: 1, w / w)$ was tested for MALDI-MSI of gangliosides in mouse brain. This IL matrix provided excellent sensitivity for detection of gangliosides without significant loss of sialic-acid residues, overcoming the major hurdle for imaging gangliosides in tissue using MS. Fig. 1 shows the abundance and anatomical localization of gangliosides in mouse brain using this technique [54].

ILs were used as efficient matrices in time-of-flight SIMS and MSI for cholesterol, bradykinin, 1,2-dipalmi- toyl-sn-glycero-3-phosphocholine, and 1,2-dipalmitoylsn-glycero-3-phosphoethanolamine. Using two ILs derived from $\alpha$-cyano-4-hydroxycinnamic acid, the molecular ion intensities of the targets were greatly improved. ILs were demonstrated to be suitable for MSI, as the IL matrices causes no changes to the sample surface by matrix crystallization or other processes, and no "hot spots" were observed in the mass spectra. in another novel application involving the matrix-enhanced MS images of onionskin membrane, fragment ions characteristic of proteins and other biomolecules observed could not otherwise have been observed in SIMS images of control samples with no matrix [55].

\subsection{Quartz-crystal microbalance}

Graphene/IL (G-IL) composites were prepared by reduction of graphene oxide (GO) to $\mathrm{G}$ in the presence of ILs in aqueous solution. The G-IL film with well-defined $\pi$ electron-rich nanospace exhibited higher affinities for aromatic compounds than that for aliphatic compounds. This could be used to selectively detect aromatics. G-IL
(A) $m / z 1546$
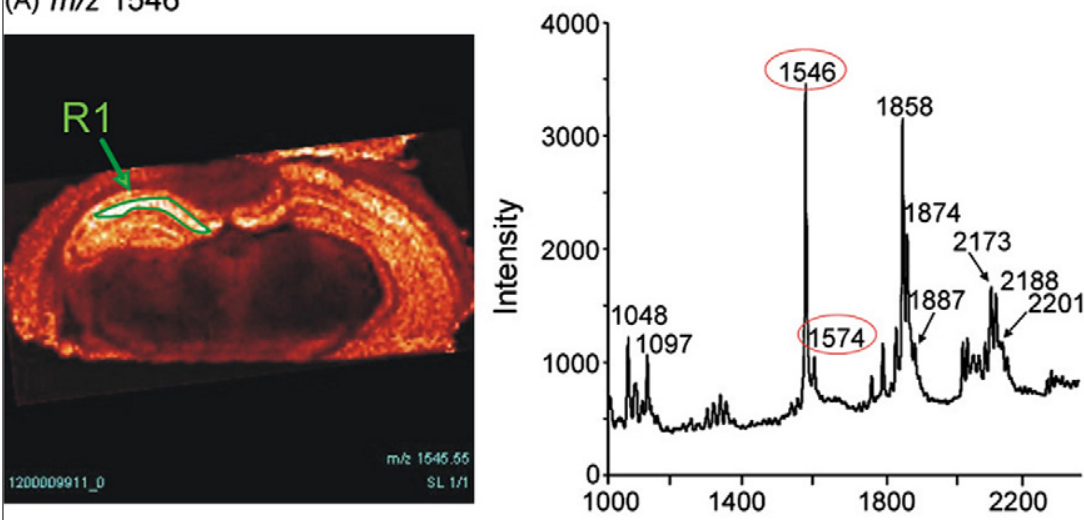

(B) $m / z 1574$

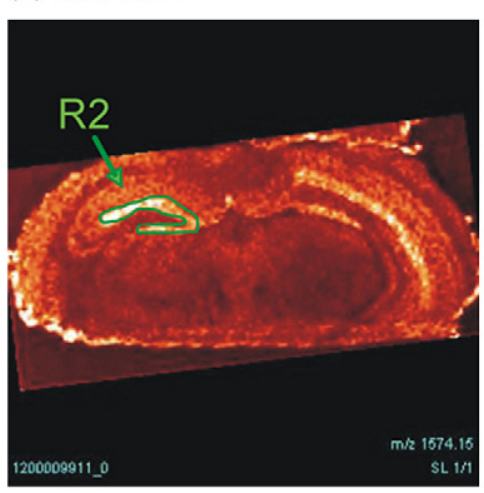

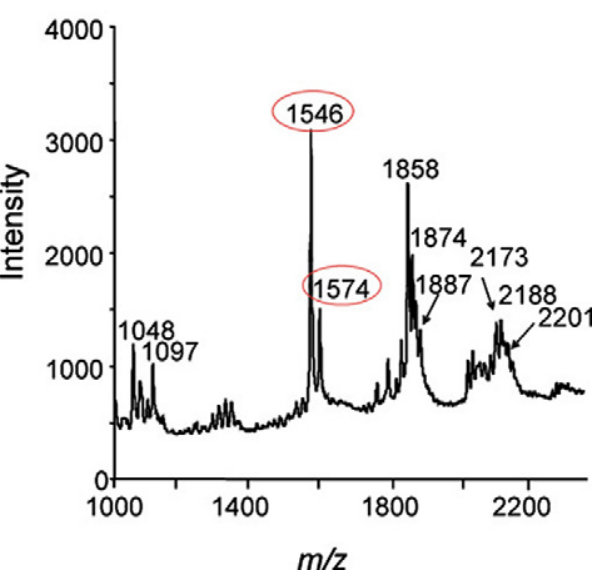

Figure 1. Imaging of mouse brain analyzed by MALDI-TOF with IL matrix composed of $\alpha$-cycno-4-hydroxy-cinnamic acid (CHCA) and 1-methylimidazole $(1: 1, \mathrm{w} / \mathrm{w})$. (A), GM1 distribution of ion at $\mathrm{m} / \mathrm{z}$ 1546. (C18:1/C18:0) and extracted mass spectra from highlighted region (CA1 region). (B) GM1 distribution at $\mathrm{m} / \mathrm{z} 1574$ (C18:1/C20:0) and extracted mass spectra from highlighted region (dentate gyrus region). (Reprinted with permission from [54], @2003 Elsevier B.V.) 
films have great potential applications in sensing trace amounts of aromatics in polluted air, according to an imaginative report [56].

Recently, an eight-sensor array developed by coupling a chemoselective IL with QCM transduction was reported to sense explosive gas with excellent sensitivity, fast response and recovery, and a workable temperature range of $27-55^{\circ} \mathrm{C}$ [57].

\section{Advances in analytical chemistry with ILs as separation medium}

ILs have been used as a separation medium in various separation techniques [e.g., GC, liquid chromatography (LC) and capillary electrophoresis (CE)]. The unique properties of ILs (i.e. low volatility, high viscosity, good thermal stability, variable polarities, and designable characteristics) offer advantages over other conventional separation medium in some separation processes.

\subsection{Simultaneous separation of polar and non-polar analytes}

In GC or LC generally, it is very hard to separate polar and non-polar molecules with the same column efficiently. Because of the unique structure of ILs, their interaction with other molecules is comprehensive, including charge interaction, chemical bonding, dipoledipole interaction, van der Waals interaction, and hydrogen bonding. These blended interactions offered ILs dual-nature properties in separation process.

Armstrong's group systematically studied the dualnature property of ILs as stationary phases in GC. Early on, they coated GC-silica capillaries with $\left[\mathrm{C}_{4} \mathrm{MIM}\right]\left[\mathrm{PF}_{6}\right]$ and $\left[\mathrm{C}_{4} \mathrm{MIM}\right] \mathrm{Cl}$ to separate compounds with different polarities. They found that an IL stationary phase demonstrated a "miraculous" dual nature, namely the IL can separate not only polar molecules like a polar stationary phase, but also non-polar molecules like a nonpolar stationary phase. After the comparing separation capacity of $\left[\mathrm{C}_{4} \mathrm{MIM}\right] \mathrm{Cl}$ and $\left[\mathrm{C}_{4} \mathrm{MIM}\right]\left[\mathrm{PF}_{6}\right]$, they found that $\left[\mathrm{C}_{4} \mathrm{MIM}\right] \mathrm{Cl}$ tended to interact more strongly with proton-donor and proton-acceptor molecules, while $\left[\mathrm{C}_{4} \mathrm{MIM}\right]\left[\mathrm{PF}_{6}\right]$ was prone to interact more strongly with non-polar solutes [58]. Further, the same group investigated the interaction of 17 ILs with probe-solute molecules using a linear free-energy approach, and confirmed that the anion had the greatest impact on the solubilizing ability and the selectivity of IL stationary phases [59].

A polar IL column was combined with a relatively non-polar commercial column (HP-5) for use in twodimensional GC. In contrast to commercial columns, the IL (trihexyl(tetradecyl) phosphonium bis(trifluoromethane)sulfonamide) column was more polar than the HP-50+ column but less polar than the DB-Wax column. The IL×HP-5 chromatogram displayed excellent separating ability for three major compound classes in diesel fuel, and the IL column kept good performance

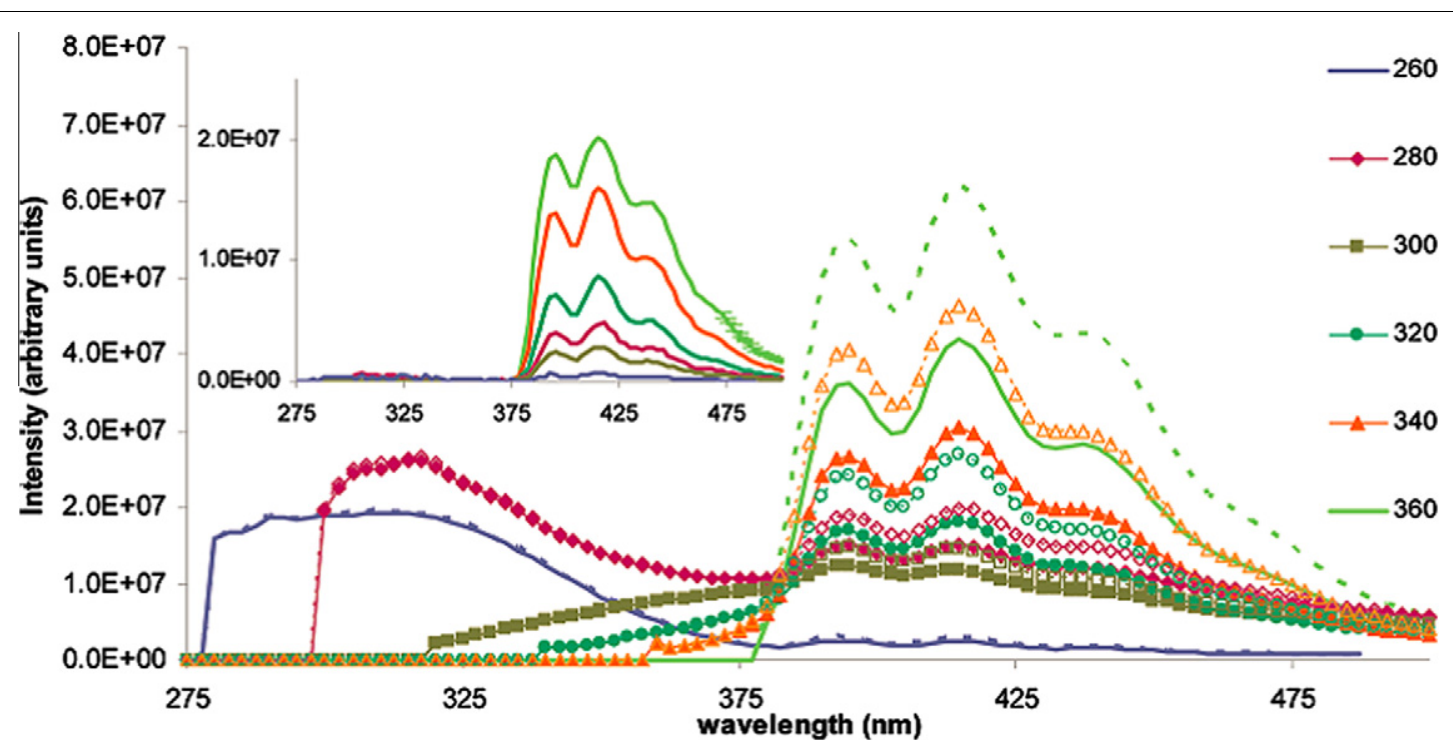

Figure 2. Emission spectra of $10 \mu \mathrm{M}$ pure enantiomeric solutions of fluorescent analytes (TFAE) in L-PheC $\mathrm{NTf}_{2}$ presented at various excitation wavelengths (260-360 nm in $20 \mathrm{~nm}$ intervals). Solid lines/filled symbols represent the S-enantiomer. Broken lines/open symbols represent the R-enantiomer. Inset: difference spectra (average of triplicate measurements; R-TFAE minus S-TFAE) for the same representative excitation wavelengths. Representative error bars for emission region 475-500 nm upon $360 \mathrm{~nm}$ excitation are included. (Reprinted with permission from [76], (C)2010 American Chemical Society.) 
while operating at temperatures of $290^{\circ} \mathrm{C}$ for several months [60]. LC columns were also used in GC $\times$ GC-MS [61], and GC $\times \mathrm{GC} \times \mathrm{GC}-\mathrm{FID}$ [62] analysis of complex mixtures.

Anderson et al. [63] reviewed retention characteristics of organic compounds using molten salt and ILs as GC stationary phases, and stressed how the dual-nature property of ILs could meet the many requirements of GC stationary phases.

The dual-nature property of ILs also enables them to enhance CE and LC separations. Depending on the ILs added, the cations/anions can coat the capillary wall and alter the electroosmotic flow of the silica capillary, while the free cations/anions in the bulk solution can associate with the analytes, and thus contribute together to the separation of target analytes [64-67]. Recently, a PIL, poly(1-vinyl-3-butylimidazolium) bromide, was used as a dynamic coating additive for CE separation of basic proteins. By adding a small amount of the PIL to the background electrolyte, the inner surface of fused-silica capillary was coated with the cationic IL, which generated a stable reversed electroosmotic flow and effectively eliminated the wall adsorption of proteins [68].

$\mathrm{He}$ et al. [69] for the first time added $\left[\mathrm{C}_{4} \mathrm{MIM}\right]\left[\mathrm{FB}_{4}\right]$ into the mobile phase for the separation of four basic compounds (i.e. the polar ephedrine and pseudoephedrine and non-polar norephedrine and methylephedrine) on a $\mathrm{C}_{18}$ column. The addition of ILs decreased band tailing, reduced band broadening, and improved resolution.

Berthod et al [70] studied the use of nine alkylmethylimidazolium ILs with different alkyl-chain lengths and anions $\left(\mathrm{Cl}^{-}, \mathrm{BF}_{4}{ }^{-}\right.$or $\left.\mathrm{PF}_{6}{ }^{-}\right)$to separate cationic basic solutes in a $\mathrm{C}_{18}$ column. It was shown that both the anion and the cation contribute to solute retention and peak efficiency. Anions can adsorb on the stationary phase surface according to their lyotropic character, and form ion pairs with the cationic basic solutes; whereas the alkyl-imidazolium cations adsorb on the $\mathrm{C}_{18}$ stationary phase through hydrophobic interaction. The cationic solute-retention factors were increased by the anion adsorption, and decreased by the cation adsorption. Anion adsorption on the stationary phase controls the cationic solute-retention factors, while cation adsorption dominates the enhancement of peak shape and efficiency.

Our group [71] tested the application of $\left[\mathrm{C}_{4} \mathrm{MIM}\right] \mathrm{Cl}$, $\left[\mathrm{C}_{8} \mathrm{MIM}\right] \mathrm{Cl}$, and $\left[\mathrm{C}_{10} \mathrm{MIM}\right] \mathrm{Cl}$ as mobile-phase additives to separate phenoxy-acid herbicides and phenols on a normal $\mathrm{C}_{18}$ silica column. Results showed that $20 \mathrm{mM}$ of $\left[\mathrm{C}_{4} \mathrm{MIM}\right] \mathrm{Cl}$ provided baseline separation and symmetrical chromatographic peaks.

Recently, imidazolium-based ILs were used as modifiers to separate a group of basic $\beta$-blockers. Sodium dodecyl sulfate and $\left[\mathrm{C}_{4} \mathrm{MIM}\right]\left[\mathrm{PF}_{6}\right]$-modified mobile phases showed high retention to $\beta$-blockers, due to the strong association of the cationic solutes with the anionic surfactant and $\left[\mathrm{PF}_{6}\right]^{-}$adsorbed on the stationary phase. By contrast, the behaviors of $\left[\mathrm{C}_{6} \mathrm{MIM}\right]\left[\mathrm{BF}_{4}\right]$ and triethylamine are similar, as the cations of these additives are significantly adsorbed on the stationary phase, whereas the corresponding anions are weakly, or not, adsorbed [72].

\subsection{Enantiomeric separations}

To achieve enantiomeric separations by GC and LC, the stationary liquids are usually synthesized by time-consuming and labor-intensive procedures consisting of multi-step reactions [73]. Enantiomeric separation can be achieved using enantiomeric stationary liquids prepared by dissolving a chiral selector into an achiral IL, or synthesis of chiral ILs. As a class of designable solvents, chiral ILs can be fabricated by varying cations, anions or both cations and anions in the same IL.

Enantiomeric separation was accomplished in GC with chiral IL stationary liquids [74]. Three N,N-dimethylephedrinium-based chiral ILs were fabricated and coated on fused-silica capillary column to generate a new chiral stationary phase. Chiral alcohols (including diols), chiral sulfoxides, chiral epoxides, and acetylated amines appeared to be well separated by the N,N-dimethylephedrinium-based ILs.

By treating 6-tosyl- $\beta$-cyclodextrin with 1,2-dimethylimidazole or 1-amino-1,2,3-triazole, four IL-functionalized $\beta$-cyclodextrins ( $\beta$-CDs) were prepared and bonded to silica gel to obtain chiral stationary phases for HPLC separation of 16 chiral aromatic alcohol derivatives and two racemic drugs [75].

In a recent report, Bwambok et al. [76] originally synthesized a multifunctional IL, L-phenylalanine ethyl ester bis(trifluoromethane) sulfonimide (L-PheC ${ }_{2}-\mathrm{NTf}_{2}$ ), to serve as solvent, chiral selector, and fluorescent report at the same time in chiral analytical measurements of different analytes including fluorescent and non-fluorescent compounds. Fig. 2 shows typical emission spectra of pure enantiomeric solutions of fluorescent analytes (TFAE) in $\mathrm{L}-\mathrm{PheC}_{2} \mathrm{NTf}_{2}$ collected at representative excitation wavelengths.

Except for IL-based GC and LC, IL-based SLME was also coupled with lipase reactions to extract (S)-ibuprofen selectively from a racemic mixture [77].

\section{Conclusion}

By exploiting the unique properties of ILs, great advances in analytical chemistry have been achieved, and more advances are expected in most sub-disciplines of analytical chemistry (e.g., sample preparation, separation and detection). Functional ILs (e.g., magnetic ILs) can reasonably be expected to be applied in sample preparation. 
The combination of ILs with nanomaterials has great potential in most analytical fields. Given the large pool of ILs, more task-specific ILs will hopefully be synthesized for various specific applications (e.g., enantiomeric separation). With commercially-available IL-based GC columns, more and more successes in separation of complex mixture will be forthcoming.

The successful application of ILs in analytical chemistry strongly depends on overcoming some obstacles (e.g., the impurity, the instability and the toxicity of some ILs), and more efforts are needed to resolve these problems.

\section{Acknowledgement}

This work was supported by the National Science Fund for Distinguished Young Scholars (21025729), the National Natural Science Foundation of China (20921063), and the China Postdoctoral Science Foundation (No. 2011M500411).

\section{References}

[1] T. Welton, Chem. Rev. 99 (1999) 2071.

[2] R. Liu, J.F. Liu, Y.G. Yin, X.L. Hu, G.B. Jiang, Anal. Bioanal. Chem. 393 (2009) 871.

[3] P. Sun, D.W. Armstrong, Anal. Chim. Acta 661 (2010) 1.

[4] A.J. Carmichael, K.R. Seddon, J. Phys. Org. Chem. 13 (2000) 591.

[5] D.E. Armstrong, J.L. Anderson, G.Z. Wei, Anal. Chem. 78 (2006) 2893.

[6] J.P. Hallett, T. Welton, Chem. Rev. 111 (2011) 3508.

[7] Q.H. Zhang, S.G. Zhang, Y.Q. Deng, Green Chem. 13 (2011) 2619.

[8] N.V. Plechkova, K.R. Seddon, Chem. Soc. Rev. 37 (2008) 123.

[9] J.F. Liu, J.Å. Jönsson, G.B. Jiang, Trends Anal. Chem. 24 (2005) 20.

[10] S. Pandey, Anal. Chim. Acta 556 (2006) 38.

[11] T.D. Ho, A.J. Canestraro, J.L. Anderson, Anal. Chim. Acta 695 (2011) 18

[12] A. Berthod, M. Ruiz-Ángel, S. Carda-Borch, J. Chromatogr., A 1184 (2008) 6.

[13] M.P. Marszałł, R. Kaliszan, Crit. Rev. Anal. Chem. 37 (2007) 127.

[14] M. Koel, Crit. Rev. Anal. Chem. 35 (2005) 177.

[15] S.R. Liu, P.K. Dasgupta, Anal. Chem. 67 (1995) 2042.

[16] J.F. Liu, G.B. Jiang, Y.G. Chi, Y.Q. Cai, Q.X. Zhou, J.T. Hu, Anal. Chem. 75 (2003) 5870.

[17] J.F. Peng, J.F. Liu, G.B. Jiang, C. Tai, M.J. Huang, J. Chromatogr., A 1072 (2005) 3.

[18] E. Aguilera-Heirador, R. Lucena, S. Cárdenas, M. Valcárcel, Anal. Chem. 80 (2008) 793.

[19] C. Yao, W.R. Pitner, J.L. Anderson, Anal. Chem. 81 (2009) 5054.

[20] S. Pedersen-Bjergaard, K.E. Rasmussen, Anal. Chem. 71 (1999) 2650.

[21] J.F. Peng, J.F. Liu, X.L. Hu, G.B. Jiang, J. Chromatogr., A 1139 (2007) 165.

[22] Y. Tao, J.F. Liu, X.L. Hu, H.C. Li, T. Wang, G.B. Jiang, J. Chromatogr., A 1216 (2009) 6259.

[23] M. Rezaee, Y. Assadi, M.R.M. Hosseini, E. Aghaee, F. Ahmadi, S. Berijani, J. Chromatogr., A 1116 (2006) 1.

[24] P.J. Dyson, D.J. Ellis, T. Welton, Can. J. Chem. 79 (2001) 705.

[25] Q.X. Zhou, H.H. Bai, G.H. Xie, J.P. Xiao, J. Chromatogr., A 1177 (2008) 43.
[26] C.L. Arthur, J. Pawliszyn, Anal. Chem. 62 (1990) 2145.

[27] J.F. Liu, N. Li, G.B. Jiang, J.M. Liu, J.Å. Jönsson, M.J. Wen, J. Chromatogr., A 1066 (2005) 27.

[28] Y.N. Hsieh, P.C. Huang, I.W. Sun, T.J. Whang, C.Y. Hsu, H.H. Huang, C.H. Kuei, Anal. Chim. Acta 557 (2006) 321.

[29] F. Zhao, Y.J. Meng, J.L. Anderson, J. Chromatogr., A 1208 (2008) 1.

[30] Y.J. Meng, V. Pino, J.L. Anderson, Anal. Chem. 81 (2009) 7107.

[31] Q.C. Zhao, J.C. Wajert, J.L. Anderson, Anal. Chem. 82 (2010) 707 .

[32] E. Wanigasekara, S. Perera, J.A. Crank, L. Sidisky, R. Shirey, A. Berthod, D.W. Armstrong, Anal. Bioanal. Chem. 396 (2010) 511.

[33] Y. He, J. Pohl, R. Engel, L. Rothman, M. Thomas, J. Chromatogr., A 1216 (2009) 4824.

[34] L. Pang, J.F. Liu, J. Chromatogr., A 1230 (2012) 8.

[35] S. Carda-Broch, A. Berthod, D.W. Armstrong, Anal. Bioanal. Chem. 375 (2003) 191.

[36] K. Shimojo, N. Kamiya, F. Tani, H. Naganawa, Y. Naruta, M. Goto, Anal. Chem. 78 (2006) 7735.

[37] F. Kubota, Y. Koyanagi, K. Nakashima, K. Shimojo, N. Kamiya, M. Goto, Solvent Extract. Res. Dev. 14 (2007) 115.

[38] F. Tang, Q.L. Zhang, D.D. Ren, Z. Nie, Q.A. Liu, S.Z. Yao, J. Chromatogr., A 1217 (2010) 4669.

[39] A.E. Visser, R.P. Swatloski, W.M. Reichert, R. Mayton, S. Sheff, A. Wierzbicki, J.H. Davis, R.D. Rogers, Environ. Sci. Technol. 36 (2002) 2523.

[40] J.H. Olivier, F. Camerel, R. Ziessel, Chem. Eur. J. 17 (2011) 9113.

[41] J.M. Reyna-Gonzalez, A.A.J. Torriero, A.I. Siriwardana, I.M. Burgar, Anal. Chem. 82 (2010) 7691.

[42] E.D. Bates, R.D. Mayton, T. Ntai, J.H. Davis, J. Am. Chem. Soc. 124 (2002) 926.

[43] Q.C. Zhao, J.L. Anderson, J. Chromatogr., A 1217 (2010) 4517.

[44] J. Hoffmann, M. Nuchter, B. Ondruschka, P. Wasserscheid, Green Chem. 5 (2003) 296

[45] Y.S. Chi, Z.D. Zhang, C.P. Li, Q.S. Liu, P.F. Yan, U. WelzBiermann, Green Chem. 13 (2011) 666.

[46] F.Y. Du, X.H. Mao, G.K. Li, J. Chromatogr., A 1140 (2007) 56.

[47] V. Pino, J.L. Anderson, J.H. Ayala, V. Gonzalez, A.M. Afonso, J. Chromatogr., A 1182 (2008) 145.

[48] L. Vidal, C.E. Domini, N. Grané, E. Psillakis, A. Canals, Anal. Chem. Acta 592 (2007) 9.

[49] A. Tholey, E. Heinzle, Anal. Bioanal. Chem. 386 (2006) 24.

[50] D.W. Armstrong, L.K. Zhang, L.F. He, M.L. Gross, Anal. Chem. 73 (2001) 3679.

[51] J.A. Crank, D.W. Armstrong, J. Am. Soc. Mass. Spectrom. 20 (2009) 1790.

[52] A. Berthod, J.A. Crank, K.L. Rundlett, D.W. Armstrong, Rapid Commun. Mass. Spectrom. 23 (2009) 3409.

[53] S. Shimma, Y. Sugiura, T. Hayasaka, N. Zaima, M. Matsumoto, M. Setou, Anal. Chem. 80 (2008) 878.

[54] K. Chan, P. Lanthier, X. Liu, J.K. Sandhu, D. Stanimirovic, J.J. Li, Anal. Chem. Acta 639 (2009) 57.

[55] J.J.D. Fitzgerald, P. Kunnath, A.V. Walke, Anal. Chem. 82 (2010) 4413.

[56] Q.M. Ji, I. Honma, S.M. Paek, M. Akada, J.P. Hill, A. Vinu, K. Ariga, Angew. Chem., Int. Ed. Engl. 49 (2010) 9737.

[57] A. Rehman, A. Hamilton, A. Chung, G.A. Baker, Z. Wang, X.Q. Zeng, Anal. Chem. 83 (2011) 7823.

[58] D.W. Armstrong, L. He, Y.S. Liu, Anal. Chem. 71 (1999) 3873.

[59] D.W. Armstrong, J.L. Anderson, J. Ding, T. Welton, J. Am. Chem. Soc. 124 (2002) 14247.

[60] J.V. Seeley, S.K. Seele, E.K. Libby, Z.S. Breitbach, D.W. Armstrong, Anal. Bioanal. Chem. 390 (2008) 323.

[61] G. Purcaro, P.Q. Tranchida, C. Ragonese, L. Conte, P. Dugo, G. Dugo, L. Mondello, Anal. Chem. 82 (2010) 8583.

[62] W.C. Siegler, J.A. Crank, D.W. Armstrong, R.E. Synovec, J. Chromatogr., A 1217 (2010) 3144. 
[63] C. Yao, J.L. Anderson, J. Chromatogr., A 1216 (2009) 1658.

[64] M. Vaher, M. Koel, M. Kaljurand, Electrophoresis 23 (2002) 426.

[65] E.G. Yanes, S.R. Gratz, M.J. Baldwin, S.E. Robison, A.M. Stalcup, Anal. Chem. 73 (2001) 3838.

[66] T.F. Jiang, Y.L. Gu, B. Liang, J.B. Li, Y.P. Shi, Q.Y. Ou, Anal. Chim. Acta 479 (2003) 249.

[67] W.D. Qin, S.F.Y. Li, Electrophoresis 23 (2002) 4110.

[68] J. Li, H.F. Han, Q. Wang, X. Liu, S.X. Jiang, Anal. Chem. Acta 674 (2010) 243.

[69] L.J. He, W.Z. Zhang, L. Zhao, X. Liu, S.X. Jiang, J. Chromatogr., A 1007 (2003) 39

[70] A. Berthod, M.J. Ruiz-Angel, S. Huguet, Anal. Chem. 77 (2005) 4071.
[71] X.L. Hu, J.F. Peng, Y.J. Huang, D.Q. Yin, J.F. Liu, J. Sep. Sci. 32 (2009) 4126.

[72] J.J. Fernández-Navarro, M.C. García-Álvarez-Coque, M.J. RuizÁngel, J. Chromatogr., A 1218 (2011) 398.

[73] C. Ravelet, E. Peyrin, J. Sep. Sci. 29 (2006) 1322.

[74] J. Ding, T. Welton, D.W. Armstrong, Anal. Chem. 76 (2004) 6819.

[75] Z.M. Zhou, X. Li, X.P. Chen, X.Y. Hao, Anal. Chem. Acta 678 (2010) 208

[76] D.K. Bwambok, S.K. Challa, M. Lowry, I.M. Warner, Anal. Chem. 82 (2010) 5028.

[77] E. Miyako, T. Maruyama, N. Kamiya, M. Goto, Chem. Commun. (2003) 2926. 